“C 2019 IEEE. Personal use of this material is permitted. Permission from IEEE must be obtained for all other uses, in any current or future media, including reprinting/republishing this material for advertising or promotional purposes, creating new collective works, for resale or redistribution to servers or lists, or reuse of any copyrighted component of this work in other works." 


\section{GENERAL INSTRUCTION}

- Author: We cannot accept new source files as corrections for your paper. If possible, please annotate the PDF proof we have sent you with your corrections and upload it via the Author Gateway. Alternatively, you may send us your corrections in list format. You may also upload revised graphics via the Author Gateway.

- Author: Please confirm the name of the corresponding author.

- Authors are required to pay a page charge of $\$ 150.00$ per page for any regular paper that exceeds 8 pages.

There is no fee for the first 8 pages. If you cannot pay these page charges please advise us.

- Page limits: No regular paper may exceed 12 typeset pages. Short papers must not exceed 4 pages.

\section{QUERIES}

Q1. Author: Please confirm or add details for any funding or financial support for the research of this article.

Q2. Author: Please provide the postal codes for the affiliations of the authors M. Gheisarnezhad and N. Vafamand.

Q3. Author: Please provide the expansions of PI/PID and SISO.

Q4. Author: Fig. 8 is not cited in the text. Please cite it at its appropriate place in the text.

Q5. Author: Please provide the department in Ref. [1].

Q6. Author: Please update Refs. [38]-[40].

Q7. Author: Please update and provide the years in [42]-[44]. 


\title{
Robust Frequency Regulation in Mobile Microgrids: HIL Implementation
}

\author{
Mohammad-Hassan Khooban ${ }^{\circledR}$, Senior Member, IEEE, Meysam Gheisarnezhad, \\ Navid Vafamand ${ }^{(0)}$, Mohammad Jafari ${ }^{\circledR}$, Member, IEEE, Saleh Mobayen ${ }^{(0)}$, \\ Tomislav Dragicevic (ㄹ) Senior Member, IEEE, and Jalil Boudjadar ${ }^{\left({ }^{\circ}\right.}$
}

Abstract-It is undeniable that marine vessel systems play an important role to transfer huge loads and weapons with low cost. However, ship power systems produce a lot of greenhouse gases, which in turn lead to serious environmental pollution. Hence, the utilizing of wind turbines (WTs), solar generation, sea wave energy (SWE), and energy storage systems (ESSs) in marine vessel power systems have been attracting a lot of attention in recent years. In this paper, it is assumed that a marine vessel power system with photovoltaic (PV), WT, SWE, and ESS can be regarded as a mobile-islanded MG. Then, a novel topology for hybrid shipboard microgrids (MGs) is presented. Next, in order to make a balance between consumption and power generation in shipboard MGs, an optimal modified model-free nonlinear sliding mode controller is introduced for the secondary load frequency control. Since the quality of the control actions of the proposed model-free approach depends on its parameters, a hybrid version of the sine-cosine algorithm (SCA) and wavelet-mutation (WM), called SCAWM, is employed to find the best value of these coefficients. Comparisons are conducted with other existing methodologies, such as model predictive control, interval type-2 fuzzy logic controller, and conventional PI (PI) to establish the supremacy of the newly suggested control strategy. Finally, a real-time hardware-in-the-loop (HIL) simulation based on OPAL-RT is accomplished to affirm the applicability of the suggested controller, from a systemic perspective, for the load frequency control problem in the shipboard MG.

Index Terms-Frequency regulation, model-free nonlinear sliding mode controller (MFNSMC), on-board power grid, sine-cosine algorithm (SCA), wavelet mutation (WM).

Manuscript received June 26, 2018; revised November 8, 2018, January 17 2019, and February 16, 2019; accepted April 10, 2019. (Corresponding author: Mohammad-Hassan Khooban.)

M.-H. Khooban and J. Boudjadar are with the Department of Engineering, Aarhus University, 8200 Aarhus, Denmark (e-mail: mhkhoban@gmail.com; jalil@eng.au.dk).

M. Gheisarnezhad is with the Department of Electrical Engineering, Islamic Azad University, Najafabad Branch, Najafabad, Iran (e-mail: m.ghesar2@ gmail.com).

N. Vafamand is with the School of Electrical and Computer Engineering, Shiraz University, Shiraz, Iran (e-mail: n.vafamand@shirazu.ac.ir).

M. Jafari is with the School of Electrical and Data Engineering, University of Technology Sydney, Ultimo, NSW 2007, Australia (e-mail: mohammad. jafari@uts.edu.au).

S. Mobayen is with the Department of Electrical Engineering, University of Zanjan, Zanjan 45371-38791, Iran (e-mail: mobayen@znu.ac.ir).

T. Dragicevic is with the Department of Energy Technology, Aalborg University, 9220 Aalborg, Denmark (e-mail: tdr@et.aau.dk).

Digital Object Identifier 10.1109/JSYST.2019.2911210

\section{INTRODUCTION}

$\mathbf{O}$ VER the last decades, the management and control of the ship power systems have been a topic of intense research, mainly due to the development in the electrification technology [1]-[4]. Due to the rapid exhaustion of fossil fuels along with their environmental concerns, the potentials of renewable energy resources (RESs) such as wind turbine (WT), solar generation (SG), and sea wave energy (SWE) have been taken into account into ship power systems.

The penetration and integration of the RESs with the conventional main grid bring the frequency distraction issues that are caused by the intermittent feature of such energy resources. Technically, energy storage systems (ESSs) such as flywheels, storage batteries, and ultra-capacitors, with appropriate control, are often used in the modern grids as a good solution to offer reliable and high-quality power supply to the loads. However, the high cost of utilizing such storage elements is deterrent in establishing this scheme [5], [6].

A shipboard MG is constructed from some distributed energy resources (DERs) (or micro-sources) and local loads that are optimally planned. Up to now, the voltage and current control of the shipboard MGs are studied, whereas a few research works have been addressed in the context of the secondary frequency regulation of the shipboard MGs. For instance, a new hybrid meta-heuristic technique has been applied in [7] for the reconfiguration problem of a shipboard MG. However, the control strategy presented in [7] is not robust enough to handle the power fluctuations of the RESs. In [8], the operation of a hybrid ship power system is investigated from the economical point of view. Due to the reduction of fossil fuel consumption as well as decreasing environmental pollution, a solar generation system is applied in merchant ships in [9]. However, the proposed model in this paper is very primary and does not investigate nonlinear load models. In [10], several prevalent control methodologies have been applied to a ship power system so that a typical kind of ESSs is coordinated to smooth the $\mathrm{PV}$ production variations and to regulate the frequency and voltage fluctuations. The main weak part of the study in [10] is the controller where it is unable to meet all the intended control objectives efficiently. The power management of a shipboard power plant is addressed in [11], which uses an integrated perturbation analysis and sequential quadratic programming (IPA-SQP) algorithm. The results presented in [11] confirm the flexibility of using the suggested algorithm 
to manage the power of the considered system in various operational scenarios. In order to assess an approximation of the dynamic secure region (DSR) in shipboard MGs, a systematic technique has been proposed in [12]. Moreover, Mashayekh and Butler-Purry [12] claimed that the application of the suggested DSR evaluation technique is not only limited to all-electric ships, and further investigations can focus on implementing the technique to other forms of isolated MGs and addressing the new challenges. Besides, in [13], the load frequency control is investigated for shipboard MGs. However, the controller, which has been applied in this paper, is very fragile over uncertainties and disturbances. Hence, according to the importance of the secondary LFC to maintain the frequency of the shipboard MG system as close as possible to the nominal value, the main aim of this paper is to propose a robust and model-free controller.

Balancing of generation and load consumption, referred to as load frequency control (LFC), is one of the most prominent issues in the islanded MGs design/operation and is becoming more critical today in accordance with the increasing size, varying configuration, changing dynamics, and raising the penetration of the intermittent renewable resources. Researchers in the world have proposed a large number of LFC approaches, such as conventional PI/PID control [14], $\mathrm{H}_{\infty}$ control theory [15], noninteger control [16], [17], model predictive control (MPC) control [18], and multiagent system [19], to maintain the frequency oscillations of the MGs within an allowable range. In [20], an intelligent PI controller employing the integral square error is designed to obtain the satisfactory LFC performance of the isolated hybrid power systems. The conventional PI controllers, however, are only capable of mitigating frequency distraction in operating conditions of MGs; they cannot provide optimal control performance against the occurrence of the usual uncertainties and changes in the MG configurations. Consequently, Bevrani et al. [21] proposed an adaptive control method based on a classic PI controller for the MG frequency control in which the fuzzy logic (FL) is used to adjust the setting of the PI controller. To achieve a more robust LFC performance, the membership functions of the FL are optimally adjusted in a heuristic manner. An MPC-based plug-in hybrid electric vehicle (PHEV) is proposed in [22] to restore the frequency fluctuation of an MG effectively. The contributions of the proposed approach are as follows: first, to smooth the wind power production, and second, to decrease the number of the required PHEVs. As a new contribution to earlier research works, Pandey et al. [23] adopted the linear matrix inequalities (LMI) oriented by PSO algorithm to minimize the frequency oscillation for an integrated RESs in the forms of MG and multi-MG. The results of Pandey et al. [23] revealed that the less control effort and guaranteed robust operation is offered by the proposed LMI scheme to tackle with the various uncertainties such as wind fluctuations and load changes. In [24], a far less common method based on an infinity control is implemented for frequency oscillation damping in an autonomous MG and the approach is designed in a way that suppresses the fluctuation effects of the load, renewable resources, and dynamic perturbations. But, the sophisticated approach introduced in [24] is not feasible in many practical applications. The reason is that most of the industrial processes have complex dynamic nature and obtaining an accurate mathematical model of these systems is highly costly and in many cases impossible. Besides, small signal of a simplified hybrid energy system, including a different combination of RESs and ESSs, is presented in [25]. Lee and Wang [25] have tried to present a very simple and accurate model for the secondary LFC in MGs.

Based on what was mentioned earlier, frequency regulation in hybrid energy systems is arduous for the sake of intermittent nature of the RESs; consequently applying an appropriate control approach, to deal with these challenges, is recommended. In this regard, a new model-free adaptive controller, which uses a hybrid meta-heuristic algorithm for the optimal tuning of a sliding mode control, is designed and then devoted to the LFC of a shipboard MG. The coefficients of the sliding mode control are automatically updated based on the online measurements, by employing a hybrid SCAWA algorithm. In addition, the proposed controller covers a wide range of operating conditions without significant reduction in the system performance, which makes it a more desirable control strategy than the classic methods. The proposed method is computationally simple as opposed to the model-based schemes [22], [26] and it is a valuable feature in the practical applications. The simulation study is conducted on a shipboard MG which is configured with different renewable resources and storage devices and, subsequently, the effectiveness of the proposed controller in regulating frequency deviation is compared with MPC [22], interval type-2 fuzzy logic controller (IT2FLC) [27], and conventional PI [21]. So in brief, the contribution of this paper can be summarized as follows.

1) A novel hybrid shipboard MG topology is introduced.

2) A new load frequency controller based on adaptive tuning of the parameters of MFNSMC is presented.

3) Real data from an offshore wind farm in Sweden, solar radiation data in Aberdeen (U.K.), and SWE from the National Oceanographic Data Center are used in order to examine the performance of the proposed novel approach.

4) A multiobjective optimization based on the hybrid SCAWA algorithm is adapted to tune the parameters of the proposed model-free nonlinear controller.

5) The proposed method is validated and implemented in hardware-in-the-loop (HIL) based OPAL-RT to integrate the fidelity of the physical simulation and the flexibility of numerical simulations.

6) The novel suggested method is computationally simple and has not any complexity like the previous approaches.

The rest of this paper is arranged in the following sequence. The simplified LFC model of a complex shipboard MG is presented in Section II. Then, the proposed model-free controller based on sliding mode control is completely elaborated in Section III. A brief outline of the hybrid SCAWA algorithm is rendered in Section IV, and the multiobjective optimization problem is illustrated in Section V. The main contribution of the approach discussed in this paper is presented in Section VI. Section VII summarizes the experimental results based on OPAL-RT under various scenarios to support the functionality of the suggested control approach. Discussions and summary of results are provided in Section VIII, and finally, this paper ends with the conclusion in Section IX. 


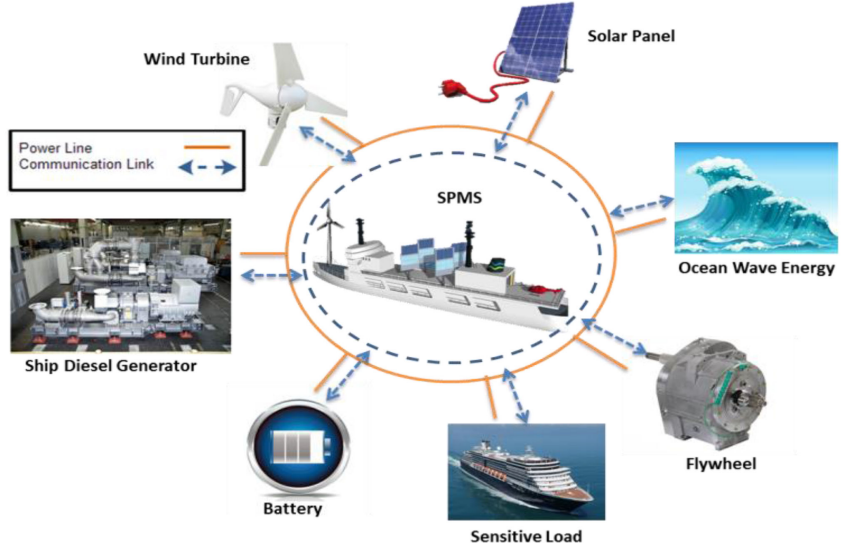

Fig. 1. Overall structure of a maritime grid.

\section{MOdELING OF A MARITIME GRID}

\section{A. Shipboard Grid Model}

Fig. 1 shows the general scheme of the shipboard MG [28] used in this paper with various distributed generation units (DGs) such as the micro-turbine, photovoltaic cell (PV), SWE and wind turbine (WT), and energy storage elements [e.g., battery energy storage system (BESS), flywheel energy storage system (FESS)]. These components can be particularly planned onboard a marine power vessel to supply its local loads connected to the shipboard MG power bus. In the hybrid power plant, the shipboard power management system (SPMS) is responsible for regulating the ship power grid and the operation of MG is controlled by the ship dispatch system (SDS). Also, the bidirectional information can be transmitted via the communication links used in the shipboard MG.

\section{B. Modeling of a Small Wind Turbine}

Wind energy is naturally intermittent; the output power of WT depends upon the wind velocity and the inherent specifications of the turbine. Based on [29], the wind velocity can be elaborated as the sum of four terms: the base wind velocity $\left(\mathrm{V}_{\mathrm{WB}}\right)$, gust wind velocity $\left(\mathrm{V}_{\mathrm{WG}}\right)$, ramp wind velocity $\left(\mathrm{V}_{\mathrm{WR}}\right)$, and noise wind velocity $\left(\mathrm{V}_{\mathrm{WN}}\right)$

$$
V_{W}=V_{\mathrm{WB}}+V_{\mathrm{WG}}+V_{\mathrm{WR}}+V_{\mathrm{WN}} .
$$

The power taken away from WT is described using the expression shown as

$$
P=\frac{1}{2} \rho_{A} A C_{P} V_{w}{ }^{3}
$$

where $A$ represents the turbine blade area, $\rho_{A}$ is the air density, $C_{P}$ is the power coefficient, and $V_{w}$ is the wind velocity. Fig. 2 shows the block diagram representation of the wind turbine that is applied as a power fluctuation source of the shipboard MG.

\section{Model of Ship Power Systems}

The diesel ship power system (DSPS) is a small-scale generating unit with some favorable properties such as quick starting

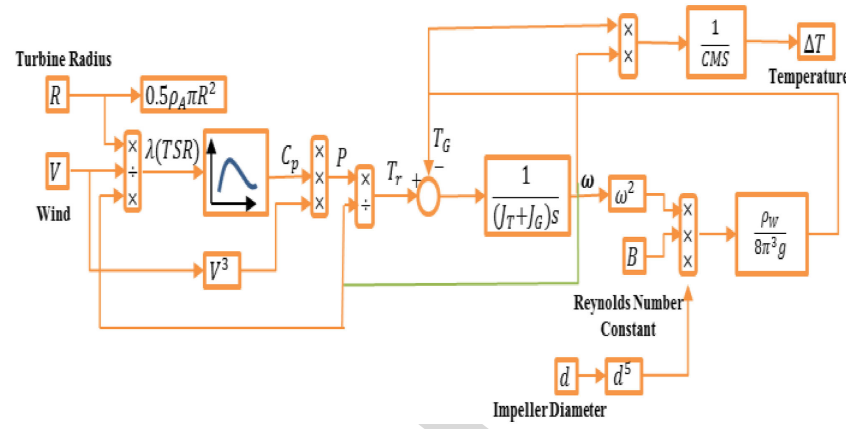

Fig. 2. Small-signal model of the wind turbine in a maritime grid.

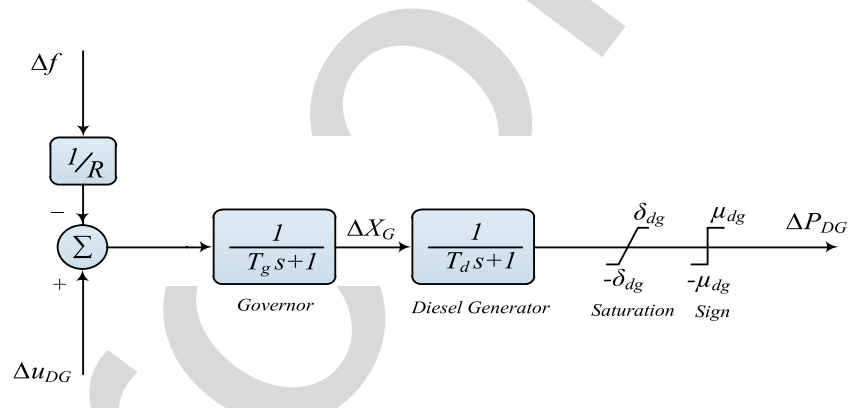

Fig. 3. Model of ship diesel power.

speed, low maintenance, and high energy efficiency. The output power of the controllable DG can be regulated to meet the load demands in a short interval. The DSPS is also able to compensate the fluctuation of the uncountable DGs embedded in the system (i.e., PV, SWE, and WT) [28]. The block diagram schematic of the DG model is displayed in Fig. 3. The model of Fig. 3 illustrates the relationship between the control signal of LFC and the DG output. As shown in Fig. 3, the components of the model including governor and generator are represented by the first-order inertia plants.

In the figure, $\Delta f$ and $\Delta u_{\mathrm{DG}}$ represent the frequency deviation and the LFC command signal from the LFC, respectively. $T_{g}$ and $T_{d}$ denote the time constant of governor and diesel generator, respectively. $\Delta X_{G}$ shows the condition of the governor's valve. The speed regulation coefficient of the DG is shown by $R$ in this figure. The $\pm \mu_{\mathrm{dg}}$ and $\pm \delta_{\mathrm{dg}}$ represent the power increment and ramp rate limits, respectively. The output power increment of the diesel power system is represented by $\Delta P_{\mathrm{DG}}$. The $\Delta P_{\mathrm{DG}}=0$ means that the demand and generation is in balance condition and there is not any need for changing the power. The $\Delta P_{\mathrm{DG}}>0$ means that the required power is higher than the actual power, whereas $\Delta P_{\mathrm{DG}}<0$ represents the condition that the actual power is less that the demand [28].

\section{Ocean Wave Energy Model}

The wave energy in oceans can be considered as a renewable energy source (RES), which is not yet fully exploited. The machine/system that turns ocean wave energy to electricity is called a wave energy converter (WEC). In this paper, a WEC is considered as an RES for shipboard MGs. The transfer function of WECs is assumed by a simple linear first-order lag by neglecting 


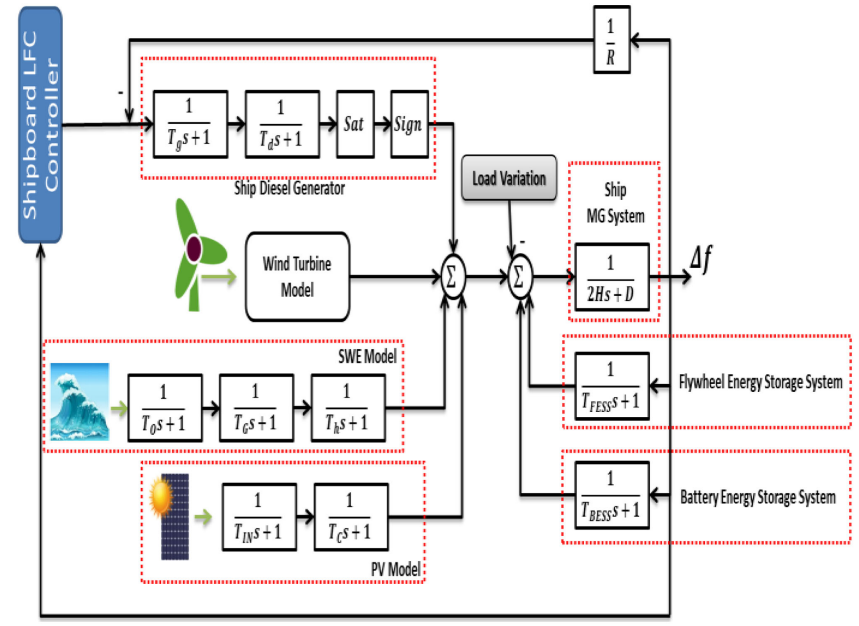

Fig. 4. Schematic of the marine vessel grid used in this paper.

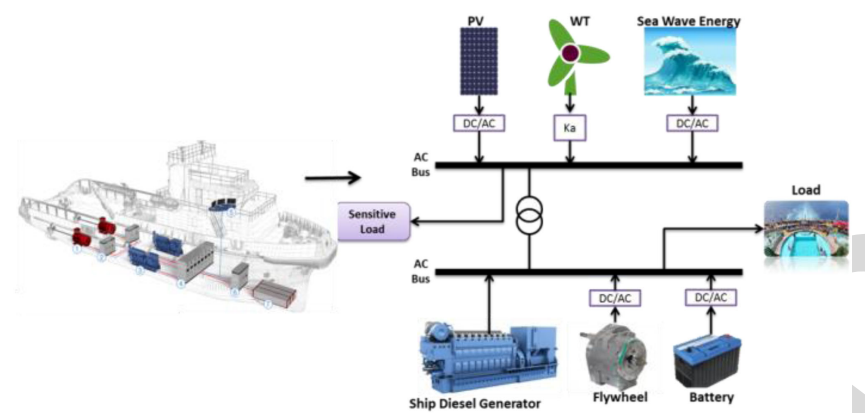

Fig. 5. General scheme for the shipboard MG with different energy sources/storage elements.

all the nonlinearities [30], [31]. The SWE governed by a WEC, which has been considered in this paper, is presented in Fig. 4.

\section{E. Solar Panels Model in a Maritime Grid}

PV cells, which are made from semiconductor materials, are able to directly convert the energy of photons to electrical energy. Due to the boundary and external contact, which are represented by a series resistor and also the small leakage current (which is represented by parallel resistance), power losses occurr. The generated power of the PV is intermittent and depends on the solar irradiance and temperature. Thus, a random power source can be used as the behavior model of PVs in simulations [21], [25]. The PV model, which is considered as a disturbance for the LFC in shipboard MG, is depicted in Fig. 4. In Fig. 4, $T_{\mathrm{IN}}$ and $T_{C}$ represent the time constant of inverter and PV, respectively.

\section{F. Common Control Approach of the LFC in a Maritime Grid}

This paper studies the LFC problem of an isolated shipboard MG that is made up of different DG components, namely DSPS, PV, WEC, and WT and storage energy devices, such as BESS and FESS. The schematic representation of the concerned shipboard MG system is sketched in Fig. 5. As shown in Fig. 5, dc/ac inverters are also installed for connecting the PV, fuel cell, BESS, and FESS to the ac bus since these components produce
TABLE I

SHIPBOARD MG PARAMETERS NOMINAL VALUES

\begin{tabular}{|c|c|c|c|}
\hline $\begin{array}{c}\text { Symbol and } \\
\text { Abbreviation }\end{array}$ & Values & Symbol and Abbreviation & Values \\
\hline $\mathbf{T}_{\mathrm{E}}$ & $2.000 \mathrm{~s}$ & $\mathbf{T}_{\mathrm{c}}$ & $0.5 \mathrm{~s}$ \\
\hline $\mathbf{T}_{\mathrm{d}}$ & $1.000 \mathrm{~s}$ & Turbine radius, $\mathrm{R}$ & $0.5 \mathrm{~m}$ \\
\hline $\mathbf{R}$ & 3.000 & Turbine height, $\mathrm{h}$ & $2 \mathrm{~m}$ \\
\hline$\delta_{\mathrm{dg}}$ & 0.010 & $\begin{array}{c}\text { Maximum of power coefficient, } \\
C_{p}\end{array}$ & 0.195 \\
\hline$\mu_{\mathrm{dg}}$ & 0.025 & Optimum STR, $\boldsymbol{\lambda}_{\mathrm{E}}$ & 0.53 \\
\hline $\mathbf{T}_{\mathrm{G}}$ & 0.5 & Turbine inertia, $\mathbf{J}_{\boldsymbol{\tau}}$ & $1.97 \mathrm{Kg} \mathrm{m}^{2}$ \\
\hline $\mathbf{T}_{\mathbf{h}}$ & 4 & Air density, $\rho_{A}$ & $1.225 \mathrm{~kg} / \mathrm{m}^{3}$ \\
\hline $\begin{array}{l}\text { D (damping } \\
\text { coefficient) }\end{array}$ & 0.012 & Water density, $\boldsymbol{\rho}_{\mathbf{w}}$ & $1000 \mathrm{Kg} / \mathrm{m}^{3}$ \\
\hline $\begin{array}{c}\text { 2H (inertia } \\
\text { constant) }\end{array}$ & 0.200 & Water specific heat, $\mathrm{C}$ & $4180 \mathrm{~J} / \mathrm{Kg} \mathrm{C}$ \\
\hline T & $0.100 \mathrm{~s}$ & Reynolds number constant, B & 5 \\
\hline $\mathrm{T}_{\text {BESS }}$ & $0.100 \mathrm{~s}$ & Heat generator inertia, JG & $1.53 \mathrm{Kg} \mathrm{m}^{2}$ \\
\hline $\mathbf{T}_{\text {In }}$ & $4.000 \mathrm{~s}$ & Impeller diameter, d & $0628 \mathrm{~m}$ \\
\hline $\begin{array}{l}\text { Mass of liquid in } \\
\text { the tank, } m\end{array}$ & $200 \mathrm{Kg}$ & "s" means Sec & \\
\hline
\end{tabular}

dc voltage. For protection issues, a circuit breaker is employed for the entire micro-sources and storage elements to disconnect them from the network. The spinning reserve for the LFC is produced by the diesel ship generator. The parameters of the concerned marine vessel system (see Fig. 5) are taken from [28] and tabulated in Table I.

The goal is to regulate the frequency of the shipboard MG such that its deviation from the desired value will be as small as possible.

\section{MODEL-FREE SMC}

\section{A. Conventional SMC}

Because of the time-variability nature of the RESs and inherent characteristic of the load, the actual model of the hybrid energy systems cannot be generated. To avoid the complexity of the mathematical modeling, the model-free techniques (e.g., fuzzy logic, neural network, etc. [21], [32]) are almost adopted for such systems. Among all the control strategies, sliding mode control is a straightforward nonlinear control method that is not sensitive to the variations in plant parameters and to nonmodeled dynamics [33]. In the following, the main concepts of SMC scheme will be introduced.

Based on [34], a SISO nonlinear system can be expressed as

$$
x^{(n)}=f(x)+g(x) u
$$

where $x \in \mathbb{R}$ is the output of the system and $u \in \mathbb{R}$ is the control input. Likewise, $f(x)$ and $g(x)$ are the nonlinear functions. Rewriting (3), one can obtain

$$
\left(\begin{array}{c}
\dot{x}_{1} \\
\vdots \\
\dot{x}_{n-1} \\
\dot{x}_{n}
\end{array}\right)=\left(\begin{array}{c}
x_{2} \\
\vdots \\
x_{n} \\
f(x)+g(x) u
\end{array}\right) \text { and } x=\left(\begin{array}{c}
\dot{x}_{1} \\
\vdots \\
\dot{x}_{n-1} \\
\dot{x}_{n}
\end{array}\right) \text {. }
$$

Let $e$ is the error signal and $x_{d}$ is the desired signal, the following equations are considered:

$$
\begin{aligned}
x_{d} & =\left(x_{d}, \dot{x}_{d}, \ddot{x}_{d}, \ldots\right) \\
e & =x-x_{d} .
\end{aligned}
$$


Generally, the SMC design of the nonlinear system can be split into two steps: first, define a proper sliding surface that enables the system to track the desired set point and, second, design the control so that force the state of the nonlinear system to the sliding surface. In this control scheme, the sliding surface is introduced as

$$
S(t)=\{x \mid s(x, t)=0\}
$$

where $s(x, t)$ is defined by

$$
s(x, t)=\left(\frac{d}{d t}+\lambda\right)^{n-1} e(t)=0, \lambda>0 .
$$

So, the error will be converged to zero exponentially on the surface. It is noted that selecting the sliding surface is somewhat arbitrary. In this step, the scheme to drive to the sliding surface is that by adding something to the $u(t)$, the states will move toward the sliding mode in finite time. Now, it is assumed that for all $x, g(x) \neq 0$. With the following condition, the control signal will be reached so that $\dot{s}=0$

$$
s=e^{(n-1)}+\cdots+\lambda^{n-1} \dot{e} .
$$

Now, by differentiating the above-stated equation

$$
\begin{aligned}
\dot{s} & =e^{(n)}+\cdots+\lambda^{n-1} \dot{e} \\
& =x^{(n)}-x_{d}^{(n)}+\cdots+\lambda^{n-1} \dot{e} \\
& =f(x)+g(x) u-x_{d}^{(n)}+\cdots+\lambda^{n-1} \dot{e} s \\
& =e^{(n-1)}+\cdots+\lambda^{n-1} \dot{e} .
\end{aligned}
$$

The $u(t)$ can be rewritten as

$$
u^{*}=\frac{1}{g(x)}\left(-f(x)+x_{d}^{(n)}-\cdots-\lambda^{n-1} \dot{e}\right) .
$$

From (11), it is clear that if $u=u^{*}$, then all time $\dot{s}=0$. Now, in order to provide a robust SMC performance against the uncertainties, a modification is applied as

$$
u=u^{*}-\frac{k}{g(x)} \operatorname{sign}(x) .
$$

Readers are referred to [26] and [33] to learn more details about the design of the SMC scheme.

\section{B. Proposed Model-Free SMC [33]}

In this section, the initial structure of the nonlinear sliding model controller and its design scheme is presented. The performance of the MFNSMC against both uncertainties and disturbances is much better than the conventional controllers such as PID, LMI, and Lead and Lag. The outstanding feature of this scheme is that it offers a model-free specification to control the various plants and its design does not need to the model identification. The general scheme of the MFNSMC for the LFC problem of the shipboard MG is sketched in Fig. 6.

The procedure for designing the suggested model-free scheme can be summarized as follows [33].

Step 1: $\beta>0$ is a design parameter, which is selected using the experience of the controller designer. Moreover, it

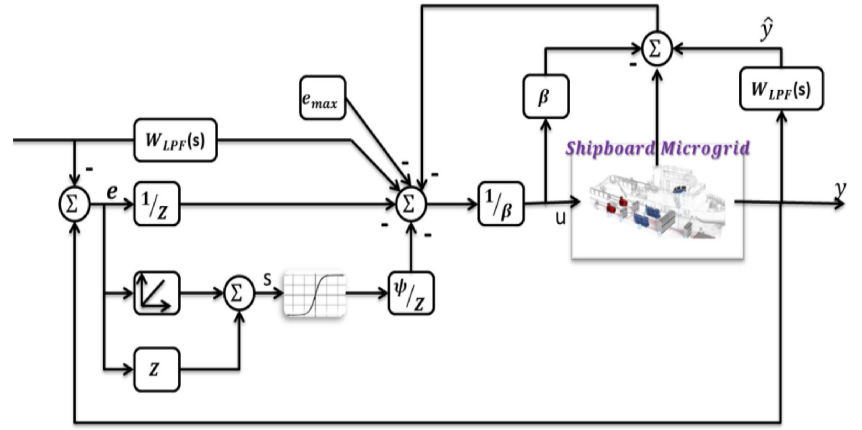

Fig. 6. Structure of the MFNSMC controller.

should be selected such that $\hat{y}$ and $\beta u$ have an equal amount.

Step 2: To implement practical derivatives of the controlled output, a first-order low-pass filter is employed with the following transfer function:

$$
W_{\mathrm{LPF}}(s)=\frac{K_{\mathrm{LPF}}}{T_{\mathrm{LPF}} S+1}
$$

where $T_{\mathrm{LPF}}$ and $K_{\mathrm{LPF}}$ are the time constant and gain of the filter, respectively. The coefficients, associated to the low-pass filter, should be chosen such that the impacts of noise and delay induced are canceled by the filter.

Step 3: Approximate a little value for $e_{\max }$.

Step 4: Adjust the coefficient $z>0$ to reach the satisfactory performance of the control technique.

Step 5: The parameter $\psi$ should be adjusted as follows:

$$
|s(t)| \psi>2 Z e_{\max }
$$

where $s(t)$ is a sliding surface. $Z>0$ determines the desired performance of the model-free controller. Moreover, $\eta>0$ is the convergence factor of the proposed controller. For sake of the stability analysis of the considered control strategy, interested readers are directed to [33].

In order to design the above-mentioned specific controller with a desirable performance, unknown control parameters must be adjusted. Tuning of these parameters is a difficult task, which is an obstacle to design the MFNSMC controller. To avoid the complexity of adjusting the parameters, a new hybrid SCAW algorithm is established.

\section{Summary OF THE ORIGINAL Sine-Cosine AlgORITHM}

The SCA is a new meta-heuristic technique that is developed based on the mathematical sine and cosine functions [35]. The algorithm employs various candidate search agents (solutions) and enables them to move toward or outward the best agent by a mathematical model of the aforesaid functions. The updating mechanism of SCA is described by the following equations:

$$
\begin{aligned}
& X_{j, t+1}= \\
& \left\{\begin{array}{l}
X_{j, t}+\omega \times \sin (\text { rand }) \times \mid \text { rand } P_{j, t}-X_{j, t} \mid ; \text { rand }<0.5 \\
X_{j, t}+\omega \times \cos (\text { rand }) \times \mid \text { rand } P_{j, t}-X_{j, t} \mid ; \text { rand } \geq 0.5
\end{array}\right.
\end{aligned}
$$


$\omega=a-t \frac{a}{T}$

where $X_{j, t}$ is the current search agent at iteration count $t$ th in the $j$ th dimension, $P_{j, t}$ is the best solution. Likewise, the control parameter of $\omega$ decreases linearly from a constant value $a$ to 0 over each course of the evolving process, where $T$ is the number of iterations.

\section{A. SCA Algorithm Based on Wavelet Mutation}

In SCA, a search agent is evolved based on the random and adaptive variables; thus, a desirable search agent cannot be found every time. Therefore, it may result in a poor optimization for some complex problems, which causes the native algorithm suffer from premature convergence, i.e., being trapped in local optima. To overcome this weakness, a hybrid variant of SCA that incorporates a wavelet-theory-based mutation mechanism named SCAWM is presented. In this way, every component of a search agent will have a chance to mutate, governed by a specified probability of $p_{w m} \in\left[\begin{array}{ll}0 & 1\end{array}\right]$. The $j$ th element of the $i$ th search agent, within the boundary $\left[L_{j}, U_{j}\right]$, will undergo mutation by a new WM operation enhanced by the sine and cos functions as proposed in the following equation:

$$
\begin{aligned}
& X_{j, t+1}= \\
& \left\{\begin{array}{l}
X_{j, t}+\sigma \times \sin (\text { rand }) \times \mid \text { rand } U_{j, t}-X_{j, t} \mid \sigma>0 \\
X_{j, t}+\sigma \times \cos (\text { rand }) \times \mid \text { rand } L_{j, t}-X_{j, t} \mid \sigma \leq 0
\end{array}\right. \\
& \sigma=\frac{1}{\sqrt{\delta}} e^{\frac{-\left(\frac{\varphi}{\delta}\right)^{2}}{2}} \cos \left(5\left(\frac{\varphi}{\delta}\right)\right)
\end{aligned}
$$

where $\delta$ is the dilation variable and $\varphi$ is randomly chosen from $[-2.5 \times a, 2.5 \times a]$.

In order to obtain a flexible mutation operator to enhance the exploration, the value of $\delta$ can be adjusted with respect to $t / T$ written as

$$
\delta=e^{-\ln (g) \times\left(1-\frac{t}{T}\right)^{\xi w m}}+\ln (g)
$$

where $\xi_{w m}$ denotes the shape variable of the monotonic increasing function, and $g$ represents the upper bound of the parameter $\delta$.

The pseudocode, as employed in the hybrid SCAWM algorithm, is illustrated in Fig. 7.

\section{MultiobJeCtive SCAWM}

Generally, the aim of designing a control problem with the multiobjective approach is to optimize multiple conflicting objective functions simultaneously in such a way that specific equality and inequalities constraints are fulfilled [16], [28].

Definition 1: A generalized multiobjective problem framework can be written as

$$
\begin{aligned}
& \text { Min }: F(x)=\left\{f_{1}(x), f_{2}(x), \ldots, f_{o}(x)\right\} \\
& \text { Subject to }:\left\{\begin{array}{l}
g_{i}(x) \geq 0 \quad i=1,2, \ldots, K_{\text {ueq }} \\
h_{i}(x)=0 \quad i=1,2, \ldots, K_{\text {eq }}
\end{array}\right.
\end{aligned}
$$

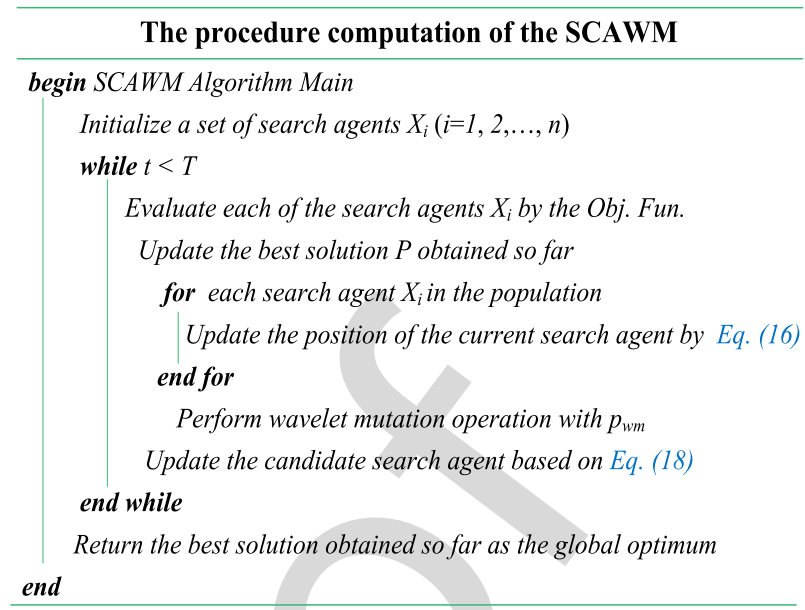

Fig. 7. Pseudocode for the suggested SCAWM algorithm.

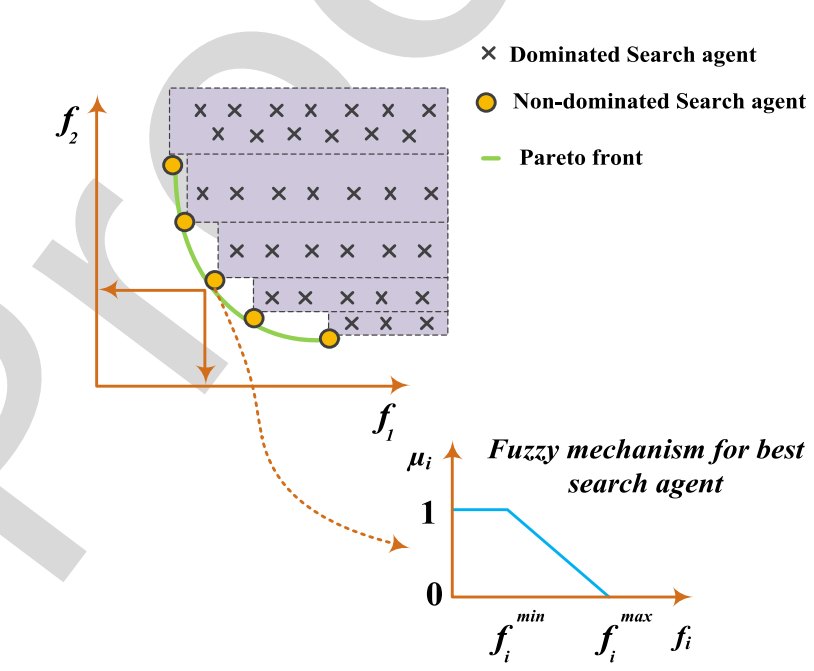

Fig. 8. Fuzzy set mechanism used for the selected Pareto set.

where $o, K_{\text {ueq }}$, and $K_{\text {eq }}$ are the number of objective functions, inequality constraints, and equality constraints, respectively. $g_{i}$ and $h_{i}$ represent the $i$ th inequality and equality constraints.

Definition 2: In an unconstrained optimization problem, an objective vector $X=\left(X_{1}, X_{2}, \ldots, X_{D}\right) \in \mathbb{R}^{D}$ dominates another objective vector $Y=\left(Y_{1}, Y_{2}, \ldots, Y_{D}\right) \in \mathbb{R}^{D}$ if the two following conditions are satisfied:

$$
\begin{aligned}
& \forall \quad j \in\{1,2, \ldots, o\}, f_{j}(X) \leq f_{j}(Y) \\
& \exists h \in\{1,2, \ldots, o\}, \quad f_{h}(X)<f_{h}(Y) .
\end{aligned}
$$

According to the above-mentioned definition, the Pareto solutions can be attained by nondominated search agents on the decisive space. Using the concept of Pareto optimization, a multiobjective version of the proposed SCAWM is applied in this study for the controller online design, i.e., tuning the coefficients embedded in the specific controller structure. For this purpose, the objective function (24) is chosen for the controller design, which comprises two contradictory objective functions $f_{1}$ and $f_{2}$
403 404 405 406 407 408 409

$$
\left.\left.\operatorname{Min}: F(x)=\left[f_{1}(x)\right), f_{2}(x)\right)\right]^{T}
$$


where

$$
\begin{aligned}
& f_{1}=\operatorname{ITSE}_{\text {set-point }}=\int_{0}^{\infty} t \cdot e_{\text {set-point }}^{2}(t) \cdot d t \\
& f_{2}=\mathrm{ISDCO}=\int_{0}^{\infty} \Delta u^{2}(t) \cdot d t
\end{aligned}
$$

where $e_{\text {set-point }}$ and $u$ represent, respectively, the error signal and the control signal produced. In the above-stated equations, $f_{1}$ is used for ensuring the good tracking of the reference set-point, whereas the $f_{2}$ tries to enhance the precision of the set-point tracking.

In order to find a better search agent among the achieved optimal solutions, the fuzzy decision-making function with a membership function (MF) is employed. In this scheme, the corresponding MF value to each objective function is determined by

$$
\begin{aligned}
& \mu_{w}^{k}(X)= \\
& \left\{\begin{array}{cl} 
& 1 f_{i}(X) \leq f_{i}^{\min }(X) \\
& 0 f_{i}(X) \leq f_{i}^{\max }(X) \\
\frac{f_{i}^{\max }(X)-f_{i}(X)}{f_{i}^{\max }(X)-f_{i}^{\min }(X)} & f_{i}^{\min }(X) \leq f_{i}(X) \leq f_{i}^{\max }(X) .
\end{array}\right.
\end{aligned}
$$

where $f_{i}^{\min }(X)$ and $f_{i}^{\max }(X)$ are the minimum and maximum values of the objective function $i$, respectively.

\section{CONTRIBUtion OF the PROPOSED APPROACH}

This paper proposes an optimal multiobjective MFNSMC scheme for the LFC problem of the shipboard MG system. The design of the suggested controller can be done by the following considerations, which have an outstanding role in its practical application.

1) The suggested hybrid nonlinear control method is straightforward and can be implemented for a reasonably different class of shipboard MGs.

2) The suggested approach can be utilized for various configurations of shipboard MGs with different components including renewable sources, storage elements, and loads.

3) The proposed controller actions are based on the available plant input/output information and can be calculated online.

4) Another benefit of the suggested control scheme is its computational efficiency, which is a valuable feature in the practical application and online control cases.

5) By using the real data, a real-time shipboard MG testbed is developed based on OPAL-RT and the performance of the MFNSMC controller is studied from a systemic perspective.

\section{HARDWARE-IN-THE-LOOP REAL-TIME SIMULATION RESULTS}

In order to verify the preeminence of the proposed modelfree technique, we develop a shipboard MG, which is depicted in Fig. 4, in MATLAB/Simulink software. The relevant parameters of the concerned system are given in Table I. The case

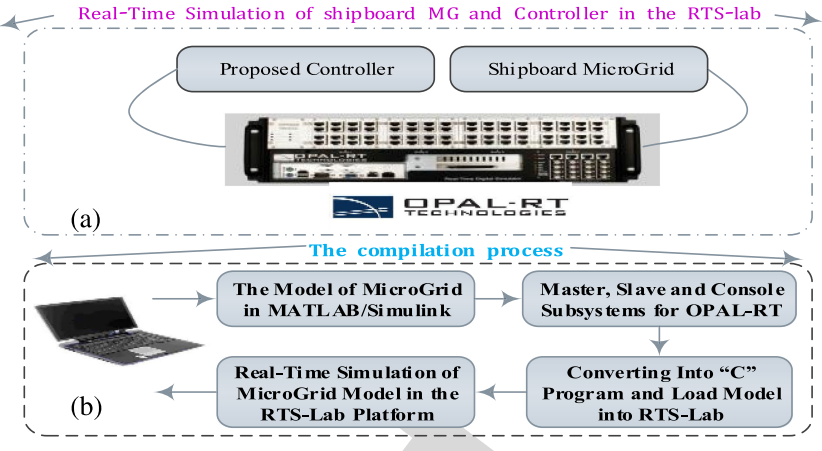

Fig. 9. OPAL RT-Lab for the validation of HIL

study is also examined with the MPC, IT2FLC, and PI controllers to verify the supremacy of the proposed controller. In order to make a fair comparison and improve the dynamic stability, the coefficients embedded in each controller structure are adjusted using the multiobjective SCAWM algorithm. In addition, the HIL simulator is established to study the applicability of the proposed scheme in the context of the shipboard MG. The HIL method provides a real-time analysis to consider errors and delays that do not exist in the offline MATLAB simulations [11], [36]. The HIL application of the proposed method was performed on a real-time simulator (RTS) wherein both the controller and plant are embedded in a single RTS [37]-[41]. The complete power system including the proposed controller was performed using the OPAL RT-Lab for the validation of HIL, as shown in Fig. 9(a). The modeling platform for the OPALRT is MATLAB/Simulink. The model-to-data workflow of the power system model under test is shown in Fig. 9(b). To make the Simulink model of the complete system including the proposed method compatible with the OPAL-RT, the model was further edited and compiled with the help of MATLAB and the OPAL RT-Lab library. After editing, the complete system model was split into three subsystems as master, slave, and console for RT-Lab simulation.

In the master subsystem, the power system model excluding the controllers and the scope was kept. The controllers were kept in the slave subsystem and the visual output devices such as scopes were kept in the console subsystem. After compilation, the complete model including all three subsystems was loaded to the OPAL-RT server for converting to the equivalent " $C$ " code of the model under test. Before simulation, the solver time step was kept in a fixed step mode, i.e., the time step in a real-time system was prespecified.

Scenario I: The shipboard MG system analysis

In the first scenario, a constant load demand, i.e., $\Delta P_{L}=0$, is considered in the isolated shipboard MG. On the other hand, the power fluctuation of WTG $\left(\Delta P_{w}\right)$, PVG $\left(\left(\Delta P_{\mathrm{pv}}\right)\right)$, and SWE $\left(\Delta P_{\mathrm{SWE}}\right)$ are used in the case study. The profile of $\Delta P_{w}$ is presented in Fig. 10(a), which is borrowed from the data of an offshore wind farm in Sweden [42], whereas Fig. 10(b) depicts the profile of $\Delta P_{\mathrm{pv}}$, which is generated from the solar radiaion data of Aberdeen (U.K.) [43]. Moreover, the curve of $\Delta P_{\mathrm{SWE}}$ is shown in Fig. 10(c) and its data have been extracted from the National Oceanographic Data Center [44]. Fig. 11 shows the 


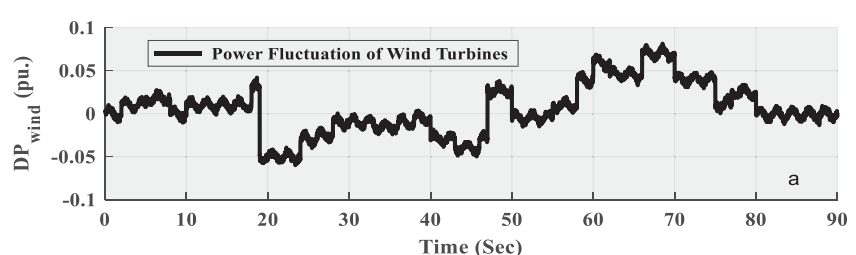

(a)

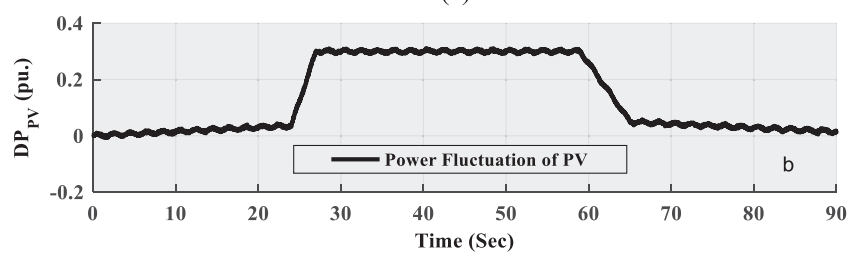

(b)

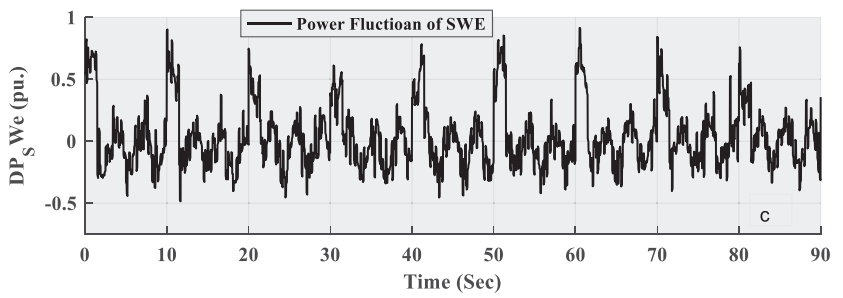

(c)

Fig. 10. Power variation. (a) WTG. (b) PV. (c) SWE.

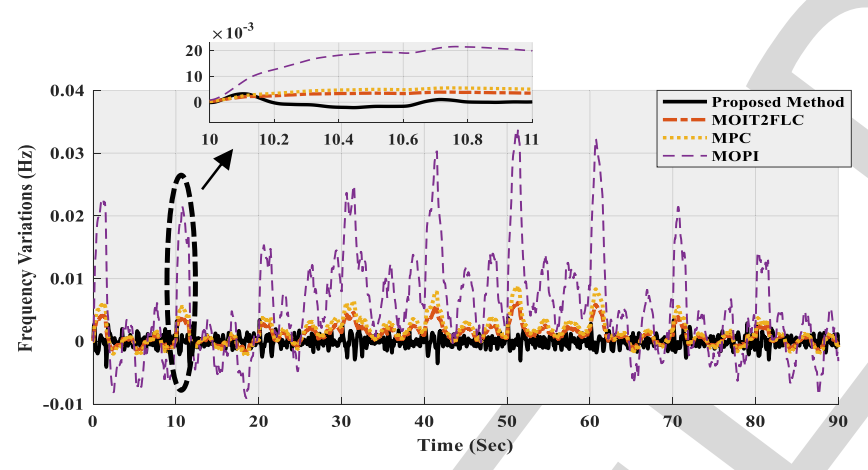

Fig. 11. Frequency deviation of the shipboard MG with WTG, PV, and SWE.

frequency deviation in the grid while using MFNSMC, MPC, MOIT2FLC, and MOPI controllers.

As shown in Fig. 11, compared with other methods, the suggested MFNSMC controller provides a considerable improvement in the transient overshoot and also with a quicker damping speed of the frequency deviation. It is also noted from Fig. 11 that using MFNSMC will yield a stable output power for shipboard MG, with less adjustment frequency and faster response, and consequently enhance the equipment life of both the storage devices and DGs. Hence, it can be inferred that by using MFNSMC controller, a more desirable qualification of the transient performance is achieved in the terms of the settling time and overshoot than the other controllers.

Scenario II: Load disturbance analysis

To ascertain the capability of the proposed technique, for an efficient LFC mechanism, against load disturbances, the case study is investigated under a multistep load variation. The profile of the load changes is demonstrated in Fig. 12, whereas the

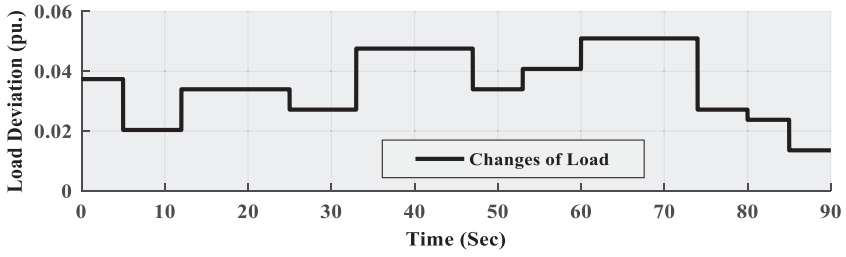

Fig. 12. Load disturbance in the maritime grid.

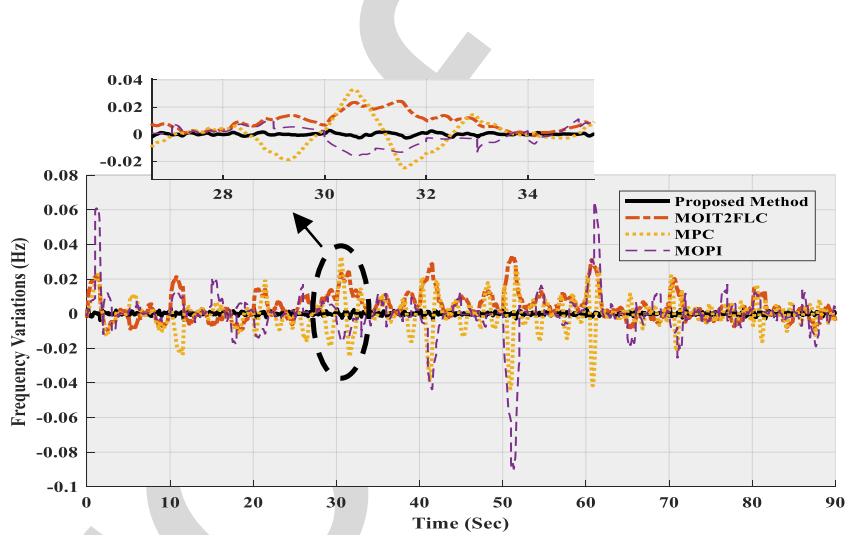

Fig. 13. Frequency deviation of the shipboard MG with WTG, PV, SWE, and load disturbances.

TABLE II

UNCERTAIN PARAMETERS OF THE SHIPBOARD MG

\begin{tabular}{|c|c|}
\hline Parameters & Variation Range \\
\hline R & $+35 \%$ \\
\hline D & $-25 \%$ \\
\hline$H$ & $+65 \%$ \\
\hline$T_{e}$ & $-25 \%$ \\
\hline$T_{g}$ & $+45 \%$ \\
\hline$T_{\text {FESS }}$ & $-35 \%$ \\
\hline$T_{B E S S}$ & $+50 \%$ \\
\hline
\end{tabular}

frequency oscillation curves of MFNSMC, MPC, MOIT2FLC, and MOPI controllers are painted in Fig. 13.

The comparative outcomes of Fig. 13 show that the MFNSMC controller, which is called for LFC mechanism, has smaller overshoot and handles the effect of the aforesaid load disturbance more effectively compared with the others. It can be seen that the frequency deviation curves have relatively larger overshoots than Scenario I. In order to assess the performance of the designed controllers in a severe condition, a large load step is applied at $t=60 \mathrm{~s}$ in the simulation. Fig. 13 confirms that in this scenario also, the suggested controller ameliorates the dynamic behavior of the frequency response having appropriate damped fluctuations and enhanced stability.

Scenario III: Robust performance analysis

For the robustness testing, the performance of the proposed controller designed in the operating condition is examined by altering some critical parameters of the shipboard MG. The percentage of the changes in the concerned system parameters is listed in Table II. According to Table II, the robustness of the 


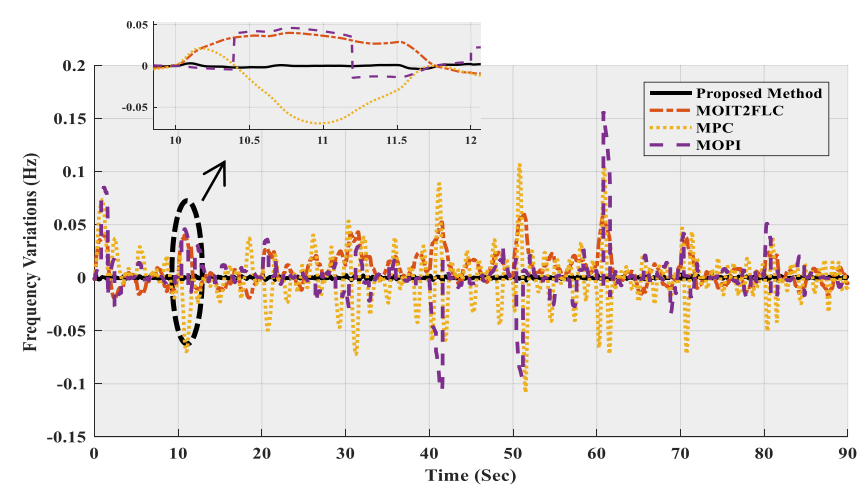

Fig. 14. Frequency response of the shipboard MG under Scenario III.

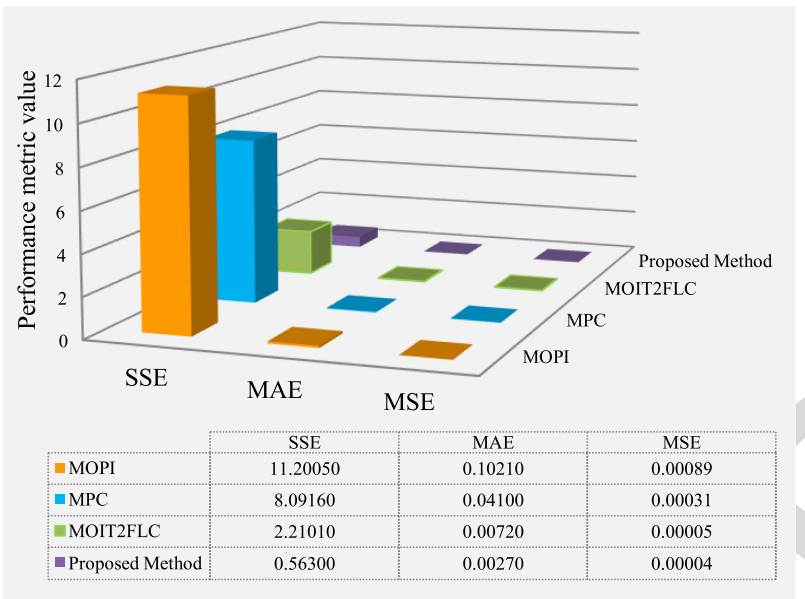

Fig. 15. Bar plot performance analysis of controllers.

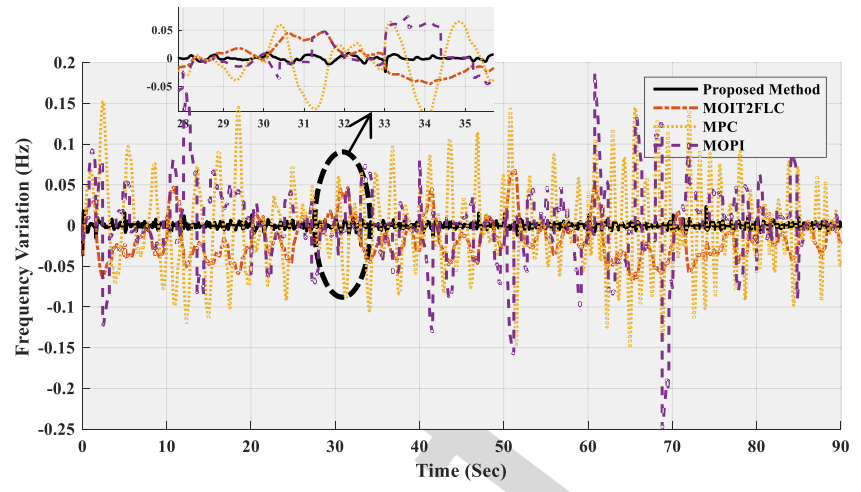

Fig. 16. Frequency response of the shipboard MG under Scenario IV.

settling time. However, these controllers are unable to ensure the stability of the system against severe uncertainties. It is also demonstrated that a high quality of the system outputs is obtainable by the MFNSMC controller. The suggested controller has a less oscillatory response and superior overshoot than the other compared controllers. Moreover, the suggested technique provides a higher level of robustness, in LFC design, to tackle parametric variations than the other controllers.

Scenario IV: Robust performance analysis against system delay

In this scenario, the effect of the delayed measuring on the performance of the uncertain shipboard MG is investigated. The considered uncertainty in this scenario is the same as scenario III, as given in Table II. Furthermore, it is assumed that the system states are measured with a constant delay $t=0.01 \mathrm{~s}$. The frequency evolutions of the closed-loop shipboard MG based on the different controllers are provided in Fig. 16.

Fig. 16 illustrates that the proposed approach is more robust against the system time delay than the other methods. Though, by comparing the results of the Scenarios III and IV, one concludes that the existence of the time delay worsens the closed-loop performance.

\section{DISCUSSIONS AND SUMMARY OF RESULTS}

The goal of this paper is to validate the applicability of the suggested mode-free technique in a real-time environment. To achieve this goal, the designed LFC controllers of the shipboard MG are simulated and examined in the OPAL-RT simulator. The OPAL-RT testbed is well known and widely used because of its high degree of fidelity to the power grid systems [11], [28]. Unlink offline simulation, the information in the testbed are transferred by the real communication infrastructures to investigate the performance of the various control methodologies in the presence of the communication latency.

In the condition that the isolated grid suffers from uncertainties and disturbances, the function of the LFC is to stabilize the grid frequency fluctuation to zero as soon as possible by regulating the input signal of the diesel ship power system. In the configured shipboard MG test system, the frequency fluctuation signal is adopted to regulate the storage devices (i.e., FESS and BESS). Thus, it eliminates the need to establish a controller for 
TABLE III

CHARACTERISTICS OF EACH SCENARIO

\begin{tabular}{|c|c|c|c|c|}
\hline & $\begin{array}{c}\text { Scenario } \\
1\end{array}$ & $\begin{array}{c}\text { Scenario } \\
2\end{array}$ & $\begin{array}{c}\text { Scenario } \\
3\end{array}$ & $\begin{array}{c}\text { Scenario } \\
4\end{array}$ \\
\hline $\begin{array}{c}\text { Variation of solar irradiation, } \\
\text { wind speed, and wave heights }\end{array}$ & $\checkmark$ & $\checkmark$ & $\checkmark$ & $\checkmark$ \\
\hline Demanding load Variation & $\mathrm{X}$ & $\checkmark$ & $\checkmark$ & $\checkmark$ \\
\hline Uncertainty & $\mathrm{X}$ & $\mathrm{X}$ & $\checkmark$ & $\checkmark$ \\
\hline Time-delay & $\mathrm{X}$ & $\mathrm{X}$ & $\mathrm{X}$ & $\checkmark$ \\
\hline
\end{tabular}

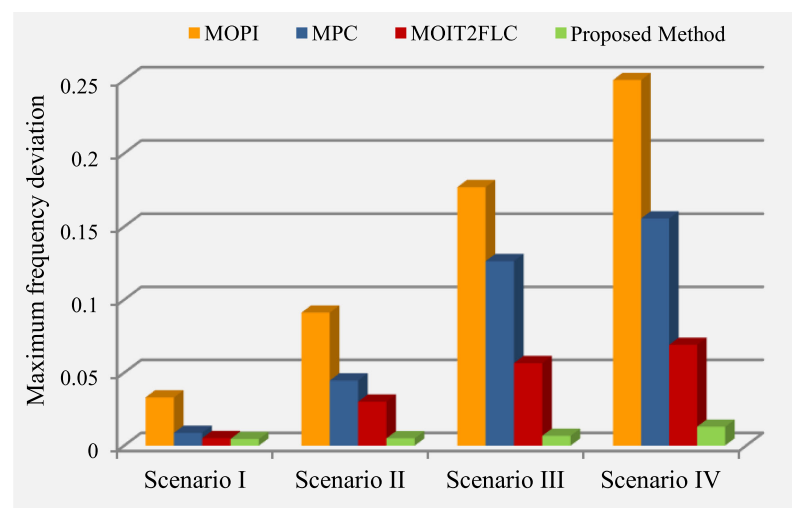

Fig. 17. Maximum value of $\Delta f$ using various control strategies.

each of the storage units in the feedback path, as opposed to the decentralized control strategies [14], and removes the need for the effective setting of each of the controllers. The dedicated control approach is efficient in practice since it decreases the costs and the additional wiring and repairs. Moreover, it obviates the need for designing the extra controllers separately, which prevents any possible deterioration in the system performance due to loop interactions.

To appraise the superiority of the suggested controller, four scenarios are considered such that each of the scenarios considers the effect of the randomness of RESs (i.e., solar irradiation, wind speed, and wave heights), the variability of demand load, changes of the MG parameters, and the presence of the time delay. Table III summarizes the characteristics of each scenario.

The experimental outcomes of the first scenario reveal that in spite of having the high plant complexity with the randomness nature of RESs, all the secondary LFC controllers can stabilize the grid frequency fluctuations but are not quite optimal. It is also observed that the performance of the designed controllers is deteriorated when the other severe conditions (e.g., the variability of load demand, parametric variation, and presence of time delay) are imposed to the concerned shipboard MG. By comparing the frequency fluctuations in the above-mentioned scenarios, it is simply found that the MFNSMC controller outperforms the MPC, MOPI, and MOIT2FLC controllers with smaller overshoots and smoother manner. In addition, the suggested modelfree technique takes a much shorter time to complete the full power acceleration and stabilize when encountering the system uncertainty and time delay simultaneously (scenario IV). Fig. 17 depicts a comparison of the maximum variation of $\Delta f$ using various controllers when the concerned scenarios are applied to the isolated shipboard MG. Moreover, the percentage improvement

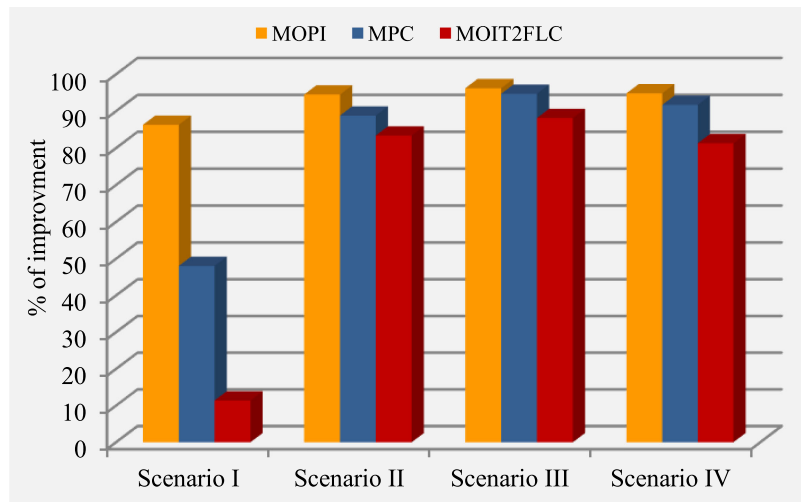

Fig. 18. Percentage improvement of suggested technique over the MOIT2FLC, MPC, and MOPI controllers.

of the model-free controller over other designed controllers is illustrated in Fig. 18. From the bar graph comparisons, it can be noted that the suggested model-free technique effectively reduces the maximum value of $\Delta f$ in comparison with the other controllers.

According to the outcomes of the aforesaid studies, the MFNSMC controller reduces the peak value of $\Delta f$ significantly, as a result, less control effort is required to stabilize the system response. It will decrease the charging/discharging of the FESS and BESS to suppress the MG frequency fluctuation, which makes possible the use of smaller storage units with longer lifetimes. This confirms that the overall energy efficiency of the system will be increased when the suggested controller is adopted to have the LFC function in the MG applications.

\section{CONCLUSION}

This paper proposes a new model-free controller to ameliorate the LFC performance of a shipboard MG case study. The control scheme discussed in this paper is less computationally exhaustive than the model-based techniques since it does not need the complexity of the mathematical modeling of the plant. In particular, the variations of the load disturbances and the fluctuation features of the renewable energies (i.e., PV, SWE, and WT) are simultaneously analyzed in the concerned hybrid power system to ascertain the efficiency of the proposed controller. Since the performance of the established MFNSMC controller highly depends on the coefficients embedded in its structure, these decisive factors are optimally adjusted by establishing a multiobjective SCAWM algorithm. Time-domain simulation outcomes have revealed that the MFNSMC controller can offer a proper tradeoff between power generation and load, and can thereby maintain the quality of power and frequency deviation within the desired limits. Moreover, the suggested controller has shown stronger robustness against a severe scenario of the changes in the system parameters. Since the design of the suggested controller is free from the system dynamic, it can be extended to various forms and configurations of the shipboard MGs. Finally, HIL simulator has been implemented to study the suitability of the suggested framework in a real-time shipboard MG testbed. The experimental simulation outcomes revealed the supremacy 
of the proposed model-free controller in comparison with the MPC, MOIT2FLC, and MOPI controllers.

\section{REFERENCES}

[1] R. Majumder, "Feasibility and challenges in microgrids for marine vessels," Ph.D. dissertation, Chalmers Univ. Technol., Gothenburg, Sweden, 2016.

[2] G. S. Misyris, A. Marinopoulos, D. I. Doukas, T. Tengner, and D. Labridis, "On battery state estimation algorithms for electric ship applications," Elect. Power Syst. Res., vol. 151, pp. 115-124, 2017.

[3] T. Van Vu, D. Gonsoulin, F. Diaz, C. S. Edrington, and T. El-Mezyani, "Predictive control for energy management in ship power systems under high-power ramp rate loads," IEEE Trans. Energy Conv., vol. 32, no. 2, pp. 788-797, Jun. 2017.

[4] J. Hou, J. Sun, and H. F. Hofmann, "Mitigating power fluctuations in electric ship propulsion with hybrid energy storage system: Design and analysis," IEEE J. Oceanic Eng., vol. 43, no. 1, pp. 93-107, Jan. 2018

[5] K. Kim, K. Park, J. Ahn, G. Roh, and K. Chun, "A study on applicability of battery energy storage system (BESS) for electric propulsion ships," in Proc. IEEE Transp. Electrif. Conf. Expo, Asia-Pac., 2016, pp. 203-207.

[6] B. Fan, C. Wang, Q. Yang, W. Liu, and G. Wang, "Performance guaranteed control of flywheel energy storage system for pulsed power load accommodation," IEEE Trans. Power Syst., vol. 33, no. 4, pp. 3994-4004, Jul. 2018.

[7] F. Shariatzadeh, N. Kumar, and A. K. Srivastava, "Optimal control algorithms for reconfiguration of shipboard microgrid distribution system using intelligent techniques," IEEE Trans. Ind. Appl., vol. 53, no. 1, pp. 474-482, Jan./Feb. 2017.

[8] G. J. Tsekouras, F. D. Kanellos, and J. Prousalidis, "Simplified method for the assessment of ship electric power systems operation cost reduction from energy storage and renewable energy sources integration," IET Elect. Syst. Transp., vol. 5, no. 2, pp. 61-69, 2014.

[9] A. Glykas, G. Papaioannou, and S. Perissakis, "Application and costbenefit analysis of solar hybrid power installation on merchant marine vessels," Ocean Eng., vol. 37, no. 7, pp. 592-602, 2010.

[10] H. Lan et al., "Modeling and stability analysis of hybrid pv/diesel/ess in ship power system," Inventions, vol. 1, no. 1, 2016, Art. no. 5.

[11] H. Park et al., "Real-time model predictive control for shipboard power management using the IPA-SQP approach," IEEE Trans. Control Syst. Technol., vol. 23, no. 6, pp. 2129-2143, Nov. 2015.

[12] S. Mashayekh and K. L. Butler-Purry, "A novel deterministic and probabilistic dynamic security assessment approach for isolated microgrids," in Proc. 19th Int. Conf. Intell. Syst. Appl. Power Syst., 2017, pp. 1-6.

[13] U. M. Reji, "Load frequency control in shipboard power systems: Design and simulation," M.S. thesis, Drexel Univ., Philadelphia, PA, USA, 2014

[14] D. C. Das, A. K. Roy, and N. Sinha, "GA based frequency controller for solar thermal-diesel-wind hybrid energy generation/energy storage system," Int. J. Elect. Power Energy Syst., vol. 43, no. 1, pp. 262-279, 2012.

[15] T. Goya et al., "Frequency control in isolated island by using parallel operated battery systems applying $\mathrm{H} \infty$ control theory based on droop characteristics," IET Renewable Power Gener., vol. 5, no. 2, pp. 160-166, 2011.

[16] I. Pan and S. Das, "Kriging based surrogate modeling for fractional order control of microgrids," IEEE Trans. Smart Grid, vol. 6, no. 1, pp. 36-44, Jan. 2015.

[17] I. Pan and S. Das, "Fractional order AGC for distributed energy resources using robust optimization," IEEE Trans. Smart Grid, vol. 7, no. 5, pp. 2175-2186, Sep. 2016.

[18] S. Kayalvizhi and D. M. V. Kumar, "Load frequency control of an isolated micro grid using fuzzy adaptive model predictive control," IEEE Access, vol. 5, pp. 16241-16251, 2017.

[19] Y. Han, K. Zhang, H. Li, E. A. A. Coelho, and J. M. Guerrero, "MAS-based Distributed coordinated control and optimization in microgrid and microgrid clusters: A comprehensive overview," IEEE Trans. Power Electron., vol. 33, no. 8, pp. 6488-6508, 2018.

[20] P. K. Ray, S. R. Mohanty, and N. Kishor, "Proportional-integral controller based small-signal analysis of hybrid distributed generation systems," Energy Convers. Manage., vol. 52, no. 4, pp. 1943-1954, 2011.
[21] H. Bevrani, F. Habibi, P. Babahajyani, M. Watanabe, and Y. Mitani, "Intelligent frequency control in an AC microgrid: Online PSO-based fuzzy tuning approach," IEEE Trans. Smart Grid, vol. 3, no. 4, pp. 1935-1944, Dec. 2012

[22] J. Pahasa and I. Ngamroo, "Coordinated control of wind turbine blade pitch angle and PHEVs using MPCs for load frequency control of microgrid," IEEE Syst. J., vol. 10, no. 1, pp. 97-105, Mar. 2016.

[23] S. K. Pandey, S. R. Mohanty, N. Kishor, and J. P. S. Catalão, "Frequency regulation in hybrid power systems using particle swarm optimization and linear matrix inequalities based robust controller design," Int. J. Elect. Power Energy Syst., vol. 63, pp. 887-900, 2014.

[24] H. Bevrani, M. R. Feizi, and S. Ataee, "Robust frequency control in an islanded microgrid: $\$\{\mathrm{H}\}$ _ $\{$ linfty $\}$ \$ and \$ $\backslash \mathrm{mu} \$$-synthesis approaches," IEEE Trans. Smart Grid, vol. 7, no. 2, pp. 706-717, Mar. 2016.

[25] D.-J. Lee and L. Wang, "Small-signal stability analysis of an autonomous hybrid renewable energy power generation/energy storage system part I Time-domain simulations," IEEE Trans. Energy Convers., vol. 23, no. 1, pp. 311-320, Mar. 2008.

[26] L. Xiong, J. Wang, X. Mi, and M. W. Khan, "Fractional order sliding mode based direct power control of grid-connected DFIG," IEEE Trans. Power Syst., vol. 33, no. 3, pp. 3087-3096, May 2018.

[27] M.-H. Khooban, T. Niknam, M. Shasadeghi, T. Dragicevic, and F. Blaabjerg, "Load frequency control in microgrids based on a stochastic noninteger controller," IEEE Trans. Sustain. Energy, vol. 9, no. 2, pp. 853861, Apr. 2018.

[28] M.-H. Khooban et al., "Shipboard microgrids: A novel approach to load frequency control," IEEE Trans. Sustain. Energy, vol. 9, no. 2, pp. 843-852, Apr. 2018.

[29] M. Katawaluwa, H. Zhang, Y. Vagapov, and J. Evans, "Simulation of wind heat generator," in Proc. IEEE Int. Conf. Electro/Inf. Technol., 2006, pp. $479-482$

[30] J. D. Nolte and R. C. Ertekin, "Wave power calculations for a wave energy conversion device connected to a drogue," J. Renewable Sustain. Energy, vol. 6 , no. 1,2014 , Art. no. 013117.

[31] J. Davidson, S. Giorgi, and J. V. Ringwood, "Linear parametric hydrodynamic models for ocean wave energy converters identified from numerical wave tank experiments," Ocean Eng., vol. 103, pp. 31-39, 2015.

[32] M. H. Khooban, T. Niknam, F. Blaabjerg, and T. Dragicevic, "A new load frequency control strategy for micro-grids with considering electrical vehicles," Elect. Power Syst. Res., vol. 143, pp. 585-598, 2017.

[33] R.-E. Precup, M.-B. Radac, R.-C. Roman, and E. M. Petriu, "Model-free sliding mode control of nonlinear systems: Algorithms and experiments," Inf. Sci., vol. 381, pp. 176-192, 2017.

[34] J.-J. E. Slotine and W. Li, Applied Nonlinear Control. Englewood Cliffs, NJ, USA: Prentice-Hall, 1991

[35] S. Mirjalili, "SCA: A sine cosine algorithm for solving optimization problems," Knowl.-Based Syst., vol. 96, pp. 120-133, 2016.

[36] M. M. Mardani, M. H. Khooban, A. Masoudian, and T. Dragicevic, "Model predictive control of dc-dc converters to mitigate the effects of pulsed power loads in naval dc microgrids," IEEE Trans. Ind. Electron., vol. 66, no. 7, pp. 5676-5685, Jul. 2019.

[37] M.-H. Khooban, "Secondary load frequency control of time-delay standalone microgrids with electric vehicles," IEEE Trans. Ind. Electron., vol. 65 , no. 9, pp. 7416-7422, Sep. 2018.

[38] M. H. Khooban, M. Gheisarnezhad, N. Vafamand, and J. Boudjadar, "Electric vehicle power propulsion system control based on time-varying fractional calculus: Implementation and experimental results," IEEE Trans. Intell. Veh., 2019, to be published, doi: 10.1109/TIV.2019.2904415.

[39] M. Gheisarnejad, M.-H. Khooban, and T. Dragicevic, "The future 5G network based secondary load frequency control in maritime microgrids," IEEE J. Emerg. Sel. Topics Power Electron., 2019, to be published, doi: 10.1109/JESTPE.2019.2898854.

[40] N. Vafamand, M. H. Khooban, T. Dragicevic, J. Boudjadar, and M H. Asemani, "Time-delayed stabilizing secondary load frequency control of shipboard microgrids," IEEE Syst. J., to be published, doi: 10.1109/JSYST.2019.2892528.

[41] M. H. Khooban, "Hardware-in-the-loop simulation for the analyzing of smart speed control in highly nonlinear hybrid electric vehicle," Trans. Inst. Meas. Control, vol. 41, no. 2, pp. 458-467, Apr. 2018.

[42] [Online]. Available: www.winddata.com. Accessed: Oct. 10, 2014.

[43] [Online]. Available: www.solargis.info/doc/solar-and-pv-data. Accessed: Oct. 10, 2014

[44] [Online]. Available: https://www.nodc.noaa.gov/General/wave.html 